\title{
Quantitative Predictions of CMB Anisotropies and Distortions
}

\author{
J. Richard Bond \\ Canadian Institute for Theoretical Astrophysics \\ University of Toronto \\ Toronto, Ontario \\ M5S 1A1, Canada
}

\begin{abstract}
The increasing precision of experiments designed to detect angular anisotropies and spectral distortions of the microwave background has now brought us near to or below the levels predicted by most theories of the formation of structure in the universe. Here I review the quantitative theoretical results for anisotropies and distortions for a wide variety of models. Many of these were presented in the Monday afternoon discussion session on the microwave background held at IAU Symposium 130. Primary and secondary anisotropies (and the associated distortions) are considered for universes with structure arising from initially Gaussian perturbations, especially the scale-invariant ones predicted by inflation, from accretion onto cosmic strings, and from shells generated by explosive energy injection, including that from superconducting strings as well as from supernovae.
\end{abstract}

\section{INTRODUCTION}

The most detailed computations of theoretical CMB radiation patterns have been made for Gaussian anisotropies generated by a Gaussian random field of density fluctuations whose amplitude still lies in the linear regime. These primary anisotropies arise (1) from Thomson scattering by flowing plasma in the early universe, (2) from fluctuations in the gravitational metric (Sachs-Wolfe effect), and (3) from fluctuations in the photon entropy (isocurvature effect). If the fluctuations are assumed to be homogeneous and isotropic as well as Gaussian, only the power spectrum for the perturbations as a function of comoving wavenumber is required to completely specify the initial conditions (apart from such parameters characterizing the unperturbed background as $H_{0}$ and the densities $\Omega$ in the various species that may populate the universe). All information about the radiation pattern on the sky is then embodied in the radiation correlation function $C(\theta) \equiv\left\langle\frac{\Delta T}{T}(\theta) \frac{\Delta T}{T}(0)\right\rangle$.

The inflationary paradigm has sharpened the range of possibilities by focussing attention on specific initial perturbation spectra, the scale invariant ones. All other spectra require a scale at an astrophysically interesting wavelength to be built into whatever the fluctuation generator would be. Although I concentrate on scale invariant spectra, a few examples of spectra which break scale invariance are considered.

Once a non-Gaussian component to the random density field develops as struc- 
Table 1: Primary Anisotropies for Primordial Gaussian Fluctuations

\section{MODEL}

OBSERVATIONS

Unbiased Adiabatic B-dom

B-dom $\Omega=\Omega_{B}=0.1$

B-dom $\Omega=\Omega_{B}=1$

Unbiased Isocurvature B-dom

B-dom ISOC $0 \Omega=1 \Omega_{B}=1$

B-dom ISOC $-1 \Omega=1 \Omega_{B}=1$

OPEN B-dom ISOC $0 \Omega=0.2 \Omega_{B}=0.2$

OPEN B-dom ISOC $-1 \Omega=0.2 \Omega_{B}=0.2$

OPEN ION B-dom ISOC $0 \Omega=0.2 \Omega_{B}=0.2$

OPEN ION B-dom ISOC - $1 \Omega=0.2 \Omega_{B}=0.2$

$\Omega=1$ biased CDM

CDM-dom $\Omega=1 \Omega_{B}=0.03$

CDM-dom $\Omega=1 \Omega_{B}=0.1$

CDM-dom $\Omega=1 \Omega_{B}=0.2$

$\mathrm{CDM}+\mathrm{B}$ hybrid $\Omega=1 \Omega_{B}=0.5$

Biased Isocurvature Axion

CDM-dom ISOC $\Omega=1 \Omega_{B} \ll \Omega$

Anti-biased Massive Neutrino $z_{n l}=1$

HOT $\left(m_{\nu}=24 \mathrm{ev}\right) \Omega=1 \Omega=0.1, b=.53$

HOT/COLD hybrid $\Omega_{\nu}=0.4 \Omega_{X}=0.5 \Omega_{B}=0.1$

\section{$\Omega<1$ Unbiased CDM}

OPEN/CDM-dom $\Omega=.2 \Omega_{X}=.17 \Omega_{B}=.03$

$\mathrm{OPEN} / \mathrm{CDM} / \mathrm{B} \Omega=.2 \Omega_{X}=.1 \Omega_{B}=.1 h=.75$

$\Lambda \neq 0$ Unbiased CDM

VAC/CDM hybrid $\Omega=1 \Omega_{v a c}=.8 \Omega_{X}=.17 \Omega_{B}=.03$

$\mathrm{VAC} / \mathrm{CDM} / \mathrm{B} \Omega=1 \Omega_{v a c}=.8 \Omega_{X}=.1 \Omega_{B}=.1 h=.75$

Non-Scale-Invariant IC's

CDM-dom + Extra Power Mountain $\Omega=1 \Omega_{B} \ll \Omega$

CDM-dom + Extra Power Plateau $\Omega=1 \Omega_{B} \ll \Omega$
Small Angle Large Angle $\begin{array}{rr}10^{6}(\Delta T / T) & 10^{6}(\Delta T / T) \\ 4.5^{\prime}\left[7.15^{\prime}\right] & 6^{\circ}\end{array}$

$<50[<15]<48$

1000

50

$\begin{array}{cc}36 & 7 \\ 28 & 14 \\ 61 & 3 \\ 84 & 16 \\ 11 & 4 \\ 14 & 31\end{array}$

$\begin{array}{cc}3\left[\begin{array}{l}5 \\ 5\end{array}\right] & 7 \\ 6 & 7 \\ 8 & 8 \\ & \end{array}$

60

20

20

8

$70[150] \quad 40$

$80[170] \quad 50$

$20[30] \quad 20$

$20[30] \quad 25$

40

60 
tures turn-around and collapse, non-Gaussian secondary anisotropies arise (4) from Thomson scattering by nonlinear bulk-flow currents, (5) from Compton cooling of inhomogeneous hot gas, and/or (6) from redshifted emission from primeval dust. The latter two mechanisms also lead to distortions in the spectrum of the CMB; dust is the most likely culprit for large distortions, such as that recently reported by the Berkeley-Nagoya group (Matsumoto et al. 1987).

Non-Gaussian primary perturbations and anisotropies may be possible in some variants of inflationary models with axions (Grinstein and Wise 1987), in cosmic string theories (e.g., Stebbins 1987), and in explosion pictures, whether supernovadriven (Hogan 1984, Vishniac and Ostriker 1985), radiation-driven (Kaiser and Hogan 1983) or driven by the electromagnetic energy from superconducting strings (Ostriker, Thompson and Witten 1987).

\section{PRIMARY GAUSSIAN ANISOTROPIES}

Sample small angle and large angle anisotropies (in units of $10^{-6}$ ) are shown in Table 1 for a variety of models of structure formation from initially Gaussian fluctuations. Unless otherwise stated, the initial fluctuation power spectrum $\mathcal{P}_{\rho}(k)$ (per decade of wavenumber) for the density in various species is taken to be scale-invariant, having $\mathcal{P}_{\rho}(k)$ proportional to $k^{3+n}$ with $n=1$ for adiabatic perturbations and $n=-3$ for isocurvature perturbations. The scale invariance for adiabatic perturbations is manifest for the gravitational potential power spectrum $\left(\mathcal{P}_{\Phi} \propto k^{-4} \mathcal{P}_{\rho}(k)\right)$.

The open, vacuum-dominated and baryon-dominated models in Table 1 are normalized assuming mass traces light, $b=1$ (unbiased); the massive neutrino models were anti-biased in the sense that the galaxies must be less clustered than the mass for the model to have gone nonlinear by redshift $z_{n l} \geq 1$ and yet still agree with the observations of the level of galaxy clustering. The rest of the models were normalized assuming the biasing factor $b=1.7$. [Technically $b=$ $\left(\Delta N_{\text {gal }} / N_{\text {gal }}\right)(\Delta M / M)^{-1}$ in a sphere of radius $10 \mathrm{~h}^{-1} \mathrm{Mpc}$ centered on a typical galaxy.] Models have $h=0.5$ unless otherwise stated $\left[H_{0} \equiv 100 h \mathrm{~km} \mathrm{~s}^{-1} \mathrm{Mpc}^{-1}\right]$. The baryon-dominated adiabatic results have been estimated from Wilson and Silk (1981). The rest of the results were obtained as part of the Bond and Efstathiou collaboration. ISOC refers to isocurvature models. The other models have adiabatic initial conditions. $\Omega_{X}$ is the density in cold dark matter (CDM), $\Omega_{\nu}$ that in massive neutrinos.

\subsection{Limits from Anisotropy Experiments}

2.1.1 Large Angle Experiments: The fiducial large angle observation for comparison with theory is taken to be the 2-beam experiment of Melchiorri et al. (1981), with the beams characterized by a full width at half maximum power of $\theta_{f w h m}=5^{\circ}$ (for a point source) switched through an angle of $\theta=6^{\circ}$. This is not an optimal design from the theoretical viewpoint, for the average CMB temperatures in the two 'illuminated' patches on the microwave sky are predicted to be highly correlated in most models. Minimizing $\theta_{f w h m}$ while maintaining $\theta$ at a few degrees is preferable for testing theories, although there are extreme experimental difficulties in such strategies. Although Melchiorri et al. reported a detection (which could be galactic emission) of an $\mathrm{rms}$ temperature difference between the locations of the two beams of $4 \times 10^{-5}$, we take their $95 \%$ confidence upper limit of $4.8 \times 10^{-5}$ as 
the bound to confront theories with.

Lasenby reported here the exciting tentative detection in the Tenerife experiment (Davies et al. 1987) of $(\Delta T / T)_{\text {rms }} \sim 4 \times 10^{-5}$ with $\theta=8^{\circ}$ and $\theta_{\text {fwhm }}=8^{\circ}$. Unfortunately, the pattern that the Tenerife beams arc out on the sky complicate the theoretical analysis of any given model: accordingly, model predictions are not presented here. Indeed the $4 \times 10^{-5}$ result is the value of $C(0)^{1 / 2}$ if $C(\theta)$ has a Gaussian profile in $\theta$, which is not the case for the scale invariant models of most interest here. However, I expect that the model predictions will not be very different from those for the Melchiorri et al. experiment, so that those amplitudes may be taken as indicative of intermediate angle values.

We have also seen at this Symposium the strong limits on anisotropy from the RELIKT experiment flown on the PROGNOZ-9 satellite given by the Soviet group (Strukov et al. 1987). For scale invariant fluctuations, they obtain a $95 \%$ confidence quadrupole limit of $C_{\ell=2}^{1 / 2}=a_{2}<2.5 \times 10^{-5}$. This result may be in conflict with the Tenerife observation for scale invariant spectra - although more analysis of the Tenerife data is required to assess whether this is a problem. Dropping the scale invariant assumption considerably relaxes the RELIKT upper limits, to $C_{\ell=2}^{1 / 2}<$ $5 \times 10^{-5}$, still a factor of two better than the upper limit obtained by Lubin and Villela (1986) using the combined Berkeley-Princeton balloon data. (The standard biased CDM model gives $C_{\ell=2}^{1 / 2}=6 \times 10^{-6}$, while the CDM+plateau model is about an order of magnitude larger.)

Strukov et al. also give a $99 \%$ confidence upper limit on the correlation function amplitude for scale invariant spectra of $\left[C\left(\theta ; \theta_{f w h m} \approx 7^{\circ}\right)\right]^{1 / 2} \lesssim 2.6 \times 10^{-5}$ over the range in $\theta$ from $20^{\circ}$ to $160^{\circ}$, with a somewhat stronger constraint at larger angles. Fixsen et al. (1983) and Lubin and Villela obtain $\left[C\left(\theta ; \theta_{f w h m} \approx 7^{\circ}\right)\right]^{1 / 2}$ $\lesssim 3.7 \times 10^{-5}$ at the $90 \%$ confidence limit, valid for $\theta \gtrsim 10^{\circ}$. These correlation function limits are typically not quite as strong theoretical constraints as those obtained by 2 -beam experiments which measure $\left[2\left(C\left(0 ; \theta_{f w h m}\right)-C\left(\theta_{j} \theta_{f w h m}\right)\right)\right]^{1 / 2}$. Thus the RELIKT experiment may not be in conflict with the Tenerife observation for unconstrained initial spectra. (Examples of such spectra might be the $n=-1$ baryon-dominated isocurvature model and the CDM+mountain model discussed below.)

2.1.2 Small Angle Experiments: At small angles, the best limit until recently was that of Uson and Wilkinson $(1984, \mathrm{UW})$ with $\theta=4.5^{\prime}$ and $\theta_{\text {fwhm }}=1.5^{\prime}$. The $95 \%$ confidence upper limit of $5 \times 10^{-5}$ quoted in Table 1 for the UW experiment is the value obtained by a maximum likelihood analysis of their data as discussed by Kaiser and Lasenby (1987). This is an increase of a factor of two over the original UW limit obtained using a different analysis method. The likelihood method is now considered superior by most workers in the subject (e.g., Wilkinson, these proceedings). The less severe UW limit allows several models we thought could safely be ruled out to now survive the $4.5^{\prime}$ test, as is evident from Table 1 .

However, a remarkably strong upper limit obtained by the Caltech Owens Valley group at $\theta=7.15^{\prime}$ with $\theta_{f w h m}=1.8^{\prime}$ now exists, as reported by Sargent at this Symposium: $(\Delta T / T)_{r m s}<1.5 \times 10^{-5}$ at the $95 \%$ confidence limit, again analyzed using the maximum likelihood technique. Some preliminary theoretical predictions for this experiment are also given in Table 1. The results quoted for this experiment are estimates obtained by neglecting beam smearing (which should be a small effect at these angular separations). 
The Caltech experiment is a 3-beam one, with the CMB temperature of the central beam subtracted from the average of the CMB temperatures in arcs of $30^{\circ} \equiv 2 \varphi_{R}$ on either side of the central beam, a distance $7.15^{\prime}$ away. The rms fluctuations are then given by

$$
\begin{gathered}
\left\langle(\Delta T / T)^{2}\right\rangle=2\left(C_{F}(0)-C_{F}(\varpi)\right)-\frac{1}{2}\left(C_{F}(0)-C_{F}(2 \varpi)\right) \\
-\int_{0}^{\varphi_{R}} \frac{d \varphi}{\varphi_{R}}\left(1-\frac{\varphi}{\varphi_{R}}\right)\left[\left(C_{F}(0)-C_{F}(2 \varpi \sin \varphi)\right)+\left(C_{F}(2 \varpi)-C_{F}(2 \varpi \cos \varphi)\right)\right] \\
\varpi \equiv 2 \sin (\theta / 2), \quad \theta=7.15^{\prime}, \quad \varphi_{R}=15^{\circ} .
\end{gathered}
$$

Here, $C_{F}(\theta)$ is the radiation correlation function smoothed over the beam profile. The Uson and Wilkinson experiment was also a 3-beam experiment, but with $\varphi_{R}=$ 0 , so only eq.(1a) was required for its analysis. For the Caltech experiment, I find (1b) gives relatively small corrections to (1a).

2.1.3 Very Small Angle Experiments: Interesting upper limits and possible observations of $\mathrm{rms}$ anisotropies at sub-arcminute resolution have recently been obtained with the VLA as Kellerman discussed at this meeting: $(\Delta T / T)_{r m s} \lesssim$ $2 \times 10^{-4}$ for $\theta_{f w h m} \sim 18^{\prime \prime}-60^{\prime \prime}$. The width of the last scattering surface for standard recombination models $\left(\sim 20 \mathrm{~h}^{-1} \mathrm{Mpc}\right.$ corresponding to an angle $\left.\theta_{c} \sim 10^{\prime}\right)$ is sufficiently large that primary anisotropies are effectively erased on angular scales less than the coherence angle $\theta_{c} \gg 1^{\prime}$ : the VLA observations may tell us about secondary anisotropies (§3) but not about primary ones.

\subsection{Constraints on Theoretical Models}

- Adiabatic baryon-dominated models violate the small angle limits. Although the $\Omega_{B}=1$ result appears marginally consistent with the UW constraint, it will undoubtedly fail to satisfy the Caltech $7.15^{\prime}$ limit by a large margin.

- The classic baryon dominated isocurvature models with initial spectral index $n=0$ (Poisson seed model) or $n=-1$ (phenomenological) that were very popular in the 1970s have been computed by Peebles (1987) and Efstathiou and Bond (1987); the quoted results were taken from the latter work. The models are labelled B-dom ISOC $n$ if there is normal recombination and by ION B-dom ISOC $n$ if the universe remains fully ionized as might be expected in these models since collapse on the smallest scales should occur shortly after recombination - if not before. The surprisingly large anisotropies on small scales for reionized models given in Table 1 are due to second order effects discussed in $§ 3.2$.

These initial fluctuation spectra are not scale invariant and there are no realistic proposals for generating them in the early universe with the required amplitudes to reproduce observed cosmic structure. If there were an inflationary mechanism giving isocurvature baryon perturbations, it would probably give scale invariant $n=-3$ fluctuations, which can be ruled out on the basis of large angle anisotropies, which are even larger than those for the isocurvature CDM models of Table 1.

- The isocurvature axion model, with the axions forming the cold dark matter, can be ruled out by large angle anisotropies, even with biased galaxy formation. The large values are due to the isocurvature effect in which the CMB fluctuations 
on scales much larger than the horizon size at recombination $\left(\sim 2^{\circ}\right.$, corresponding to $\sim 200 \mathrm{~h}^{-1} \mathrm{Mpc}$ ) is just proportional to the scale invariant initial fluctuations in the photon entropy per CDM particle. By contrast, the small angle fluctuations are not very large. For example, in an $h=0.75$ model, with the rest of the parameters as indicated in Table 1 for the biased isocurvature axion model, $\Delta T / T$ is $2 \times 10^{-6}$ and $4 \times 10^{-6}$ for the UW and Caltech experiments respectively.

- The standard $\Omega=1$ adiabatic CDM models with biased galaxy formation (Davis et al. 1985, Bardeen et al. 1986) are not in conflict with any of the observations, even for $\Omega_{B}$ as high as 0.5 , unless we suppose the Tenerife experiment is seeing primary CMB anisotropies.

- Open CDM-dominated models are ruled out even by the renormalized UW experiment and, very strongly, by the Caltech results. Primack presented UW limits for CDM models with a range of values of $\Omega_{B}$. Where they overlap with our calculations, they agree.

- Vacuum-dominated CDM models with $\Omega_{v a c} \approx 0.8$ are now largely ruled out by the Caltech experiment. Invoking a nonzero cosmological constant, parameterized here by $\Omega_{v a c} \equiv \Lambda /\left(3 H_{0}^{2}\right)$, is one of the few ways to make models with low densities in CDM and baryons - which have nice large scale structure properties (Bardeen, Bond and Efstathiou 1987, Blumenthal, Dekel and Primack 1987) compatible with inflation. If we raise $h$ to 0.75 in the VAC/CDM hybrid model of Table 1, survival of the model becomes marginal: we get $9 \times 10^{-6}$ and $1.7 \times 10^{-5}$ for the UW and Caltech experiments without beam smearing.

- Massive neutrino models even with $z_{n l}$ as low as one will apparently be ruled out by the Caltech experiment - although the calculation remains to be done.

- Biased CDM models with broken scale invariance can give large intermediate angle anisotropies. Scale invariant adiabatic spectra - predicted in the standard inflationary scenario - have constant power in the gravitational potential fluctuations per decade of wavelength. The last two models of Table 1 modify this scale invariant initial spectrum by adding to the flat piece a $k^{-2}$ power law ramp beginning at cluster scale, $k^{-1} \sim 5 \mathrm{~h}^{-1} \mathrm{Mpc}$, rising until $k^{-1} \sim 200 \mathrm{~h}^{-1} \mathrm{Mpc}$, where the power either crashes to the old flat value (mountain) or flattens at the new large height (plateau).

These initial spectra are designed so that the biased CDM model agrees with the reported observations of the $\sim 600 \mathrm{~km} \mathrm{~s}^{-1}$ large scale streaming velocities of regions about $50 \mathrm{~h}^{-1} \mathrm{Mpc}$ across (Dressler et al. 1987), of the cluster-cluster correlation function (Bahcall and Soneira 1983) and the cluster-galaxy correlation function (Seldner and Peebles 1977, but see Lilje and Efstathiou 1987 who get smaller clustering).

Mountains of extra power cannot be obtained with scale invariant initial conditions no matter what the modification of the constituents of the universe: plateaus are required.

Scale invariance can be broken in inflation models with more than one weakly coupled scalar field (Kofman and Linde 1987 [KL], Silk and Turner 1987, Bardeen, Bond and Salopek 1987 [BBS]). Mountains are also difficult to obtain in perturbation generation models by quantum fluctuations during inflation (BBS, but see KL). 
Although BBS showed that initial fluctuation spectra with the ramp plus plateau shape are generic in inflation with more than one scalar field, the tuning of the initial amplitude of the last scalar to drive inflation and of the potential parameters to be just right so that the ramp in the power spectrum (1) coincides with cluster scale, (2) doesn't rise too high to violate the microwave anisotropy constraints, yet (3) rises high enough to give the cluster correlations, requires a miraculous set of circumstances. We already demand one miracle from inflation models, a potential just flat enough to get the fluctuations exactly as required for structure formation. The large scale observations may demand a second miracle.

The Tenerife observation is tantalizingly similar to the anisotropies predicted for these extra-power spectra. The CDM+plateau predictions at larger angular scales would, however, be in contradiction to the low upper limits for flat fluctuation spectra reported for the RELIKT experiment. Since mountains seem so unlikely, this multiple scalar field solution may already be disproved. What seems clear in any case is that inferring much large scale power from clustering and velocity flow evidence must necessarily imply large intermediate angle microwave background anisotropies for Gaussian fluctuations.

\section{DISTORTIONS AND SECONDARY ANISOTROPIES}

\subsection{Normal Cosmic Strings}

Stebbins (1987) has discussed the various mechanisms by which cosmic strings would generate anisotropy, including primary effects associated with the string network and secondary effects associated with matter accretion. There is a characteristic scale of $\Delta T / T$ expected for loops of string of tension (and mass per unit length) $\mu$ and size $R$ : The gravitational metric perturbation is $h \sim 2 G(2 \pi \mu R) / R \sim 4 \pi G \mu$. The metric changes by $\Delta h \sim h$ in a light crossing time across the loop as the string flops around relativistically, continually overshooting in its attempts to straighten out. CMB radiation crossing the string is therefore shifted in temperature by the time changing gravitational field by an amount $\Delta T / T \sim \Delta h \sim 4 \pi G \mu, \sim 10^{-5}$ for the currently preferred value of the string tension, $\mu \sim\left(10^{16} \mathrm{GeV}\right)^{2}$, i.e., $G \mu \sim 10^{-6}$, as predicted by grand unified theories and as required to match structure formation. Stebbins has analyzed this process in detail and finds $(\Delta T / T)_{r m s} \sim$ $(3-10) G \mu$.

This same $G \mu$ scaling arises for one of the more spectacular mechanisms for $\Delta T / T$ generation suggested by Kaiser and Stebbins (1984). A long straight string has a metric which locally looks like flat space, but is in fact a conical space with a wedge of angle $8 \pi G \mu$ taken out. If the string moves across the sky with speed $v$, a sharp temperature discontinuity occurs of magnitude $\Delta T / T \sim 8 \pi G \mu v\left(1-v^{2}\right)^{-1 / 2}$, again $\sim 10^{-5}$ for grand unified strings. Observation of such a discontinuity would be a unique signal of the presence of cosmic strings.

Gravitational metric fluctuations also arise from matter responding to the mass of the loops and long strings. The perturbed matter ultimately dominates $h$, inducing an ordinary Sachs-Wolfe effect for the clustering mass which also scales as $G \mu$ : Traschen, Turok and Brandenberger (1986) estimate values of $5 \times 10^{-7}$ at $0.5^{\circ}$ and $3 \times 10^{-6}$ at $6^{\circ}$ for the fractional temperature difference without beam smearing from loops produced before recombination. As well there will be Sunyaev Zeldovich anisotropies from the hot gas that accreted onto loops defining the sites 
of rich clusters.

Although one can catalogue all possible effects generating anisotropy in the cosmic string scenario, calculating the statistical distribution of anisotropy from a realistic string network for direct comparison with experiments has so far proved intractable.

\subsection{The Vishniac Effect $-\Delta T / T$ to Quadratic Order}

It is conventional wisdom that early reionization of the universe erases small angle anisotropies. A universe that never recombines has a very fuzzy decoupling surface (of last scattering), with a width corresponding to $\theta_{c} \sim 5^{\circ}$, compared with the value $\theta_{c} \sim 10^{\prime}$ of standard recombination. Thomson scattering from troughs and crests of the velocity field destructively interfere on scales smaller than the decoupling width $\left(\Delta T / T \propto \int \sigma_{T} \bar{n}_{e} v_{e} d t\right.$, with $\sigma_{T}$ the Thomson cross section and $\bar{n}_{e}$ the average electron density).

However, as Vishniac (1987) has pointed out, significant small scale anisotropy can develop from quadratic nonlinearities in the scattering $\left(\propto \int \sigma_{T} \delta n_{e} v_{e} d t\right)$ which do not suffer from destructive interference, as different wave-modes are coupled. He has calculated this effect for CDM and HOT DM models, assuming they reionize early enough (which is unlikely according to Bond and Efstathiou 1984): for the UW experiment, he obtains $10^{-6}$ for the biased CDM model and $(1-2) \times 10^{-5}$ for the massive neutrino model with $z_{n l}=4$, a model which can be ruled out on other grounds (\$3.5).

Reionization is likely to occur early enough in the baryon-dominated isocurvature models with the steep $n=0$ and $n=-1$ initial spectra. Efstathiou and Bond (1987) have computed the magnitude of the Vishniac effect for these models, and the results are included in the $\Delta T / T$ values quoted in the ionized models of Table 1. It will be interesting to see how close the theoretical values come to the Caltech limit.

\subsection{The Berkeley-Nagoya Distortion}

Matsumoto et al. $(1987 ; \mathrm{BN})$ report a distortion on the Wein side of the CMB. The excess energy $u_{d i s t}$ in the distortion relative to the total CMB energy $u_{c m b}$ is:

$\begin{array}{ccccccc}\lambda & 480 & 709 & 1160 & \text { dust(tot) } & \text { Compton (tot) } & \text { X-decay (tot) } \\ u_{d i s t}(\lambda) / u_{c m b}(\lambda) & 3 & 0.7 & 0.1 & 0.2 & 0.06 & 0.2\end{array}$

This table also gives an approximate expression for the amount of excess radiation relative to that in the $\mathrm{CMB}$ required by the three main mechanisms proposed to explain the distortion:

(1) Primeval dust emission: Bond, Carr and Hogan (1986 [BCH1], 1987 [BCH2]) showed that if galaxies (even dwarfs) exist at $z \sim 10$ then they cover the sky, and if they are dust-laden then all energy from the near IR to the $\mathrm{X}$ gets absorbed and re-emitted in the far IR, with a peak wavelength $\lambda \sim 700 \mu$ which is relatively model insensitive. In $\mathrm{BCH} 2$ we apply our theory to the BN data. Preliminary results imply three surpising features are required: (a) very large energy outputs, $\sim 20 \%$ of the CMB, difficult to arrange for conventional metal-producing stars; (b) large optical depths to dust implying a high redshift of dust formation and/or abundant production in dwarf proto-galaxies (e.g., the biased CDM model may 
not form dwarfs early enough); and (c) a redshifted dust temperature near to the CMB temperature, $T_{d} / T_{c m b} \sim 1.4$. Associated intensity anisotropies might be at the percent level in the far IR and measurable at ground based facilities (§3.4).

(2) Compton Cooling: Compton upscattering off hot plasma electrons dumps a fraction $4 y$ extra energy into the CMB, where $y=\int n_{e} \sigma_{T}\left(T_{e} / m_{e}\right) d t$ is the Compton $y$-parameter. The best fit $y$-parameter found by $\mathrm{BCH} 2$ is 0.016 , although the fit is not great at short $\lambda$. All late time energy injection models (e.g., galaxy explosions) that could produce such a large $\bar{y}$ would be expected to generate large (unobserved) Rayleigh Jeans (RJ) anisotropies, $\Delta T / T \approx-2 \Delta y$.

Thus it is best to have high redshift energy injection from a large number $N$ of small scale sources along any given line of sight since $\Delta y$ from $1 / \sqrt{N}$ fluctuations would be small, especially with beam smearing. Nuclear or accretion energy from stars or black holes is not effective since it goes into radiation which, once the medium is ionized, propagates relatively freely to us. Although most of the heat pumped into the electrons by the ionization process Compton cools into the CMB, the energy is likely to be small compared with the direct redshifted radiation.

Early non-radiative energy injection does not suffer from this problem. One of the most intriguing possibilities for this is superconducting cosmic strings (Ostriker, Thompson and Witten 1986). Electromagnetic energy liberated below the plasma frequency of the pregalactic medium by SCS's would heat electrons which would then Compton cool, giving $\bar{y} \approx(0.3-5) \times 10^{-3} 10^{6} G \mu$ (Ostriker and Thomson 1987), provided at least $10 \%$ of the energy liberated by decaying strings is electromagnetic (with the remainder being in gravitational waves). Ostriker and Thompson show that the nature of the spectrum of string loops in the network imply RJ anisotropies, generated predominantly at late times, will not be overly large: they estimate $(\Delta T / T)_{\text {rms }} \sim 2 \times 10^{-5}$ for switching angles $\theta>4^{\prime}$ without beam smearing for $G \mu=10^{-6}$. Increasing $G \mu$ to give $\bar{y}=0.016$ may then conflict with the Caltech limit, although more precise calculations will be required.

(3) Direct Decay Radiation: K. Sato (Kowasaki and Sato 1987) showed that a weakly interacting particle $(\mathrm{X})$ of mass $\sim 5$ ev decaying with a lifetime $\sim 10^{13}$ sec can give a good fit to the BN distortion if they are about one-quarter as abundant before decay as massive neutrinos (which don't work) - provided one includes spectral modification of the decay radiation by the plasma. If one invokes dust reprocessing of the radiation by galaxies covering the sky, a much larger range of decaying particle parameters is allowed (BCH1).

\subsection{Dust Emission Anisotropies}

$\mathrm{BCH} 1$ estimated the anisotropies in the far IRB by dust emission from galaxies which just cover the sky. Bond (1987) and $\mathrm{BCH} 2$ have extended this analysis to include the high optical depth cases suggested by the BN data. Sample results for anisotropies at $\lambda=709 \mu$ are given in Table 2 for a high $(z \sim 30)$ and low $(z \sim 5$, biased CDM model) redshift of dwarf galaxy formation. The fluctuations are quite small in the RJ regime. Clustering of dwarf galaxies dominates over $\sqrt{N}$ fluctuations along the line of sight in these determinations. Unfortunately, there are rather many parameters that can be varied in the dust emission models. The $\Delta T / T$ amplitudes indicated by Table 2 are typical, although they can range up to the $\%$ level once the distortion energy exceeds that in the unperturbed CMB. 
Table 2: Secondary Anisotropies and Spectral Distortions

MODEL

$\begin{array}{cc}\left.\underset{\text { Anisotropy }}{10^{6}(\Delta T / T)_{r m s}\left(\theta_{\text {fwhm }}\right.}=24^{\prime \prime}\right) & \text { Distortion } \\ & 10^{6}\langle\Delta T / T\rangle \\ 50 & 600 \\ 3000 & 3 \times 10^{4} \\ & 10^{6} \bar{y} \\ 7 & 10 \\ 80 & 20 \\ 700 & 200 \\ 20 & 20\end{array}$

\subsection{Compton Cooling Anisotropies}

Sunyaev-Zeldovich (SZ) anisotropies arise from angular fluctuations $\Delta y$ in the $y$-parameter. Large objects such as clusters and shells from explosions tend to dominate the effect; consequently, Poisson fluctuations in the number of objects along the line of sight are more important than anisotropies due to clustering of the objects, except perhaps at degree scales if the correlation function of clusters is large.

For the calculations presented in Table 2 (Bond 1987), a simple shot noise model of structure is adopted. For example, for the biased CDM and massive neutrino models, the shots are objects (e.g., clusters) with positions specified by a random point process (peaks of Gaussian random fields with different filtering scales and initial linear density amplitudes, Bardeen et al. 1986), with shot profiles (pressure profiles) given by top hat models of virialized structures centered at each point, and with shot clustering given by terms describing $\sqrt{N}$ fluctuations and by a $\xi \sim r^{-2}$ continuous correlation function which reproduces the galaxy and cluster correlation data for CDM model parameters. (A similar technique was used for the dust anisotropies of $\S 3.4$.) The main conclusions from Table 2 are as follows:

(1) The standard biased CDM picture gives very small SZ fluctuations. The dominant contribution comes from large groups and poor clusters as opposed to rich clusters or galaxies (c.f. Ostriker and Vishniac 1986). Similar calculations by Coles and Kaiser (1987) are in basic agreement with my results. Shafer and Silk (1987) use a different assumption about the distribution of hot gas, giving clusters long extended tails, motivated by the cluster-galaxy correlation function, and also normalize to a rather high $\Delta T / T$ for the Coma cluster. They obtain values about an order of magnitude larger than those quoted here for a hierarchy similar to that for the CDM model. The CDM model might also have SZ anisotropies from pancake-like configurations (void walls) not explicitly included in any of our analyses.

A side product of this work is a triumph for the biased CDM model (with $b \approx$ 1.5 rather than the 2.1 consistently used by Davis et al. 1985): the basic structural properties and average spacings of objects ranging from dwarf galaxies through bright galaxies and groups to clusters are obtained with this simple spherical top hat collapse model and the CDM fluctuation spectrum with the biasing normalization. 
(2) Hot dark matter models with nonlinear redshifts of order 3 can be ruled out by virtue of their SZ anisotropies from very massive clusters. These neutrino clusters would also be copious X-ray emitters whose presence would have been detected by the Einstein observatory (White et al. 1984). The neutrino cluster model describes HDM universes with a high $z_{n l}$ better than the pancake model used by Szalay, Bond and Silk (1983), who estimated somewhat smaller SZ distortions, since pancakes are transitory while the clusters, representing the deepest potential wells, continue to accumulate hot gas, much of it draining from the pancakes.

(3) Explosions: SZ anisotropies from fully developed explosive bubbles are surprisingly small. For example, to generate voids of diameter $25 \mathrm{~h}^{-1} \mathrm{Mpc}$ with the rich cluster spacing $55 \mathrm{~h}^{-1} \mathrm{Mpc}$ at redshift $z_{x}=7$ requires at least $39 \mathrm{kev} / \mathrm{baryon}$, but $r m s$ SZ fluctuations and the average $y$-parameter are only $\sim 2 \times 10^{-5}$ (Table 2 ). It remains an open possibility that anisotropies arising from the earliest phases of explosion when the pressure is highly concentrated and before the structure is well developed may be large.

Acknowledgments: I thank the participants in the IAU $\Delta T / T$ discussion section and hope I have not overly misrepresented their work. The support of a Canadian Institute for Advanced Research Fellowship, a Sloan Foundation Fellowship and the NSERC of Canada is gratefully acknowledged.

\section{REFERENCES}

Bahcall, N. and Soneira, R. 1983, Ap. J., 270, 70.

Blumenthal, G., Dekel, A. and Primack, J., 1987, Ap. J., in press.

Bardeen, J.M., Bond, J.R., Kaiser, N. and Szalay, A.S. 1986, Ap. J., 304, 15.

Bardeen, J.M., Bond, J.R. and Efstathiou, G. 1987, Ap. J., 321, 28.

Bardeen, J.M., Bond, J.R. and Salopek, D.S. 1987, in Proceedings Second Canadian Conference on General Relativity and Relativistic Astrophysics, eds. A. Coley, C. Dyer (Singapore: World Scientific).

Bond, J.R., Carr, B.J. and Hogan, C.J. 1986, Ap. J. 306, 428 [BCH1]; 1987, Preprint [BCH2].

Bond, J.R. and Efstathiou, G. 1984, Ap. J. Lett. 285, L45; 1987, M.N.R.A.S. 226, 655 .

Bond, J.R. 1987, in The Early Universe, Proc. NATO Summer School, Vancouver Is., Aug. 1986, ed., Unruh, W.G. (Dordrecht:Reidel); 1987, Preprint.

Coles, S. and Kaiser, N. 1987, in preparation.

Davies, R.D., Lasenby, A.L., Watson, R.A., Daintree, E.J., Hopkins, J., Beckman, J., Sanchez-Almeida, J. and Rebolo, R. 1987, Nature 326, 462.

Davis, M., Efstathiou, G., Frenk, C.S., and White, S.D.M. 1985, Ap. J. $292,371$.

Dressler, A., Faber, S.M., Burstein, D., Davies, R.L., Lynden-Bell, D., Terlevich, R.J. and Wegner, G. 1987, Ap. J. Lett. 313, L37.

Efstathiou, G. and Bond, J.R. 1986, M.N.R.A.S. 218,103; 1987, M.N.R.A.S. 227, 33P.

Fixsen, D.J., Cheng, E.S. and Wilkinson, D.T. 1983, Phys. Rev. Lett. 50, 620.

Hogan, C.J. 1984, Ap. J. Lett. 284, L1.

Kaiser, N. and Hogan, C.J. 1983, Ap. J. 274, 7.

Kaiser, N. and Lasenby, A.L. 1987, Preprint.

Kaiser, N. and Stebbins, A. 1984, Nature 310, 391. 
Kofman, L. A. and Linde A. D. 1987, Nuc. Phys. B282, 555.

Kowasaki, M. and Sato, K. 1987, Preprint.

Lilje, P. and Efstathiou, G. 1987, Preprint.

Lubin, P. and Villela, T. 1986, in B. F. Madore and R. B. Tully, eds. Galaxy Distances and Deviations from the Hubble Flow (Dordrecht: Reidel).

Matsumoto, T., Hayakawa, S., Matsuo, H., Murakami, H., Sato, S., Lange, A.E. and Richards, P.L. 1987, Preprint [BN].

Melchiorri, F., Melchiorri, B.O., Ceccarelli, C. and Pietranera, L. 1981, Ap. J. Lett., 250, L1.

Ostriker, J.P. and Vishniac, E.T. 1986, Ap. J. Lett. 306, L51.

Ostriker, J.P., Thompson, C. and Witten, E. 1986, Phys. Lett. B180, 231.

Ostriker, J.P. and Thompson, C. 1987, Preprint.

Peebles, P.J.E., 1987, Ap. J. 277, L1.

Seldner, M. and Peebles, P.J.E. 1977, Ap. J., 215, 703.

Shafer, R. and Silk, J. 1987, in preparation.

Silk, J. I. and Turner, M. S. 1987, Phys. Rev. D35, in press.

Stebbins, A. 1987, in preparation.

Strukov, I.A., Skulachev, D.P. and Klypin, A.A. 1987, these proceedings.

Szalay, A.S., Bond, J.R. and Silk, J. 1984, Formation and Evolution of Galaxies and Large Scale Structure; The Third Moriond Astrophysics Meeting (Dordrecht: Reidel), p. 499.

Traschen, J., Turok, N. and Brandenberger, R. 1986, Phys. Rev. D34, 919.

Uson, J.M. and Wilkinson, D.T. 1984, Nature 312, 427 [UW].

Vishniac, E.T. and Ostriker, J.P. 1985, Societa Italiana di Fisica Conference Proc. $1,137$.

Vishniac, E.T. 1987, Preprint.

White, S.D.M., Frenk, C.S. and Davis, M. 1984, M.N.R.A.S. 209, 27P.

Wilson, M.L. and Silk, J. 1981, Ap. J. 243, 14. 